\title{
Long-term follow up of intractable chronic short lasting unilateral neuralgiform headache disorders treated with occipital nerve stimulation
}

\author{
Running Title: ONS for SUNCT/SUNA \\ ${ }^{1}$ Sarah Miller MBBS, ${ }^{2}$ Laurence Watkins and ${ }^{1}$ Manjit Matharu $\mathrm{PhD}$ \\ ${ }^{1}$ Headache Group, Institute of Neurology and The National Hospital for Neurology and \\ Neurosurgery, Queen Square, London, UK \\ ${ }^{2}$ Department of Neurosurgery, Institute of Neurology and The National Hospital for \\ Neurology and Neurosurgery, Queen Square, London, UK
}

Prepared For: Cephalalgia

Abstract: 201

Body: 3727

Tables: 5

Figures: 1

References: 34

Supplementary Data: 1 Table

Correspondence:

Dr MS Matharu

Senior Lecturer and Honorary Consultant Neurologist

Headache Group, Institute of Neurology and The National Hospital for Neurology and

Neurosurgery, Queen Square, London WC1N 3BG

Email: m.matharu@uclmail.net

Tel: $\quad+447595900535$

Fax: $\quad+447092120797$

Key Words: Short lasting unilateral neuralgiform headache attacks, SUNCT, SUNA, Trigeminal autonomic cephalalgia, Occipital Nerve Stimulation, Neurostimulation 


\begin{abstract}
Background

Occipital nerve stimulation is a potential treatment option for medically intractable shortlasting unilateral neuralgiform headache attacks. We present long-term outcomes in 31 patients with short-lasting unilateral neuralgiform headache attacks treated with occipital nerve stimulation in an uncontrolled open-label prospective study.
\end{abstract}

\title{
Methods
}

Thirty-one patients with intractable short-lasting unilateral neuralgiform headache attacks were treated with bilateral occipital nerve stimulation from 2007 to 2015. Data on attack characteristics, quality of life, disability and adverse events were collected. Primary endpoint was change in mean daily attack frequency at final follow-up.

\section{Results}

At a mean follow-up of 44.9 months (range 13-89) there was a 69\% improvement in attack frequency with a response rate (defined as at least a $50 \%$ improvement in daily attack frequency) of $77 \%$. Attack severity reduced by 4.7 points on the verbal rating scale and attack duration by a mean of $64 \%$. Improvements were seen in headache-related disability and depression. Adverse event rates were favorable with no electrode migration or erosion reported.

\section{Conclusion}

Occipital nerve stimulation appears to offer a safe and efficacious treatment for refractory short-lasting unilateral neuralgiform headache attacks with significant improvements 
sustained in the long-term. The procedure has a low adverse event rate when conducted in highly specialised units. 


\section{INTRODUCTION}

Short-lasting unilateral neuralgiform headache attacks (SUNHA) are a rare form of primary headache syndrome characterized by attacks of unilateral pain occurring in the trigeminal distribution. The pain is of moderate to severe intensity and is accompanied by at least one cranial autonomic symptom or sign, ipsilateral to the pain ${ }^{1}$. Attacks can last 1-600 seconds with attack frequency ranging between 1 and over 200 a day ${ }^{1,2}$. The International Classification of Headache Disorders (ICHD-3beta) describes two subsets of the syndrome defined by the presence of ipsilateral tearing and conjunctival injection: short-lasting unilateral neuralgiform headache attacks with conjunctival injection and tearing (SUNCT) (where both lacrimation and conjunctival injection are present) and short-lasting unilateral neuralgiform headache attacks with autonomic features (SUNA) (where only one of or neither of lacrimation or conjunctival injection are present) ${ }^{1}$. The chronic form of SUNHA is defined as attacks occurring for over one year with remission periods of less than one month. Seventy percent of patients suffer the chronic variant ${ }^{2}$.

First line treatment for the condition is lamotrigine but other drugs such as topiramate, oxcarbazepine and gabapentin have been reported to be effective ${ }^{3-7}$. Temporary response to lidocaine infusions and greater occipital nerve blocks have also been reported ${ }^{8-10}$. Shortlasting unilateral neuralgiform headache attacks can be difficult to treat medically and some patients prove intractable to treatment. In the past, such patients have been subject to destructive procedures of the trigeminal nerve with poor long-term outcomes ${ }^{11}$. However, recent meta-analysis suggests that microvascular decompression of the ipsilateral trigeminal nerve in selected patients with intractable SUNHA and neurovascular contact may be useful

12. Long-term outcome data from this procedure is awaited. 
Peripheral (occipital nerve stimulation) and central (ventral tegmental area deep brain stimulation) neurostimulation techniques have been carried out with some success for the syndrome. Our group have recently reported a cohort of 11 patients who have undergone deep brain stimulation for SUNHA with a median improvement in daily attack frequency of $78 \%$ with an at least $50 \%$ reduction in attack frequency seen in nine patients ${ }^{13}$. Deep brain stimulation is, however, highly invasive and associated with a small risk of fatal intracerebral hemorrhage ${ }^{14}$. Occipital nerve stimulation (ONS) appears potentially useful for chronic migraine based on a small number of randomized trials and for chronic cluster headache based on a number of open-label series ${ }^{15-22}$. Safety profile has been dominated by high lead migration and infection rates. The experience of ONS in the treatment of SUNHA is limited to a single series of nine patients ${ }^{23}$. In this prospective open-label series we report the longterm follow-up of 31 medically intractable chronic SUNHA patients treated with ONS who had tried numerous other treatment options available to them within the UK's National Health Service.

\section{METHODS}

\section{Patients}

Under the supervision of our institution's Clinical Effectiveness Supervisory Committee (CESG) with arrangements for clinical governance, consent and audit, we offered ONS to medically intractable SUNHA. The procedure was provided on the basis of a "humanitarian intervention". In addition, ethic board approval for data collection and publication was granted by Northwick Park Hospital Research Ethics Committee, Hampstead, London, UK. 
Patients were reviewed in a multidisciplinary clinic and were implanted over a period from 2007 to 2013. All patients fulfilled International Classification of Headache Disorders $2^{\text {nd }}$ edition at time of diagnosis ${ }^{24}$. Although four patients had longer duration attacks (between 15-45 minutes) than in ICHD criteria this has been described in other large clinical series and all had had some attacks less than 10 minutes during the course of the syndrome ${ }^{2}$. Patients with longer lasting attacks underwent Indometacin trials (oral or intramuscular) to rule out indometacin-sensitive headaches ${ }^{25}$. Unlike chronic cluster headache or migraine, intractability is not defined for SUNHA and so local criteria were devised. Patients were considered for ONS if they had had disabling chronic SUNHA for at least two years and had failed adequate trials of lamotrigine, topiramate, gabapentin, pregabalin and one of either carbamazepine or oxcarbazepine ${ }^{23}$. Agents were selected on the basis of reported efficacy and local experience ${ }^{2,26}$. Failed trials were defined as a lack of response, intolerable side effects or contraindications to use of medication. Microvascular decompression was not available to the patient group during the study period. Given the lack of evidence to support its use as a predictor of ONS outcome, response to greater occipital nerve block was not considered in patient selection ${ }^{27}$.

\section{Surgical Procedure}

Bilateral ONS electrodes, leads and an implantable pulse generator (IPG) were implanted in all patients (Table 1). Systems from both Medtronic $(n=26)$ and St Jude Medical $(n=5)$ were utilized with octad electrodes used in all. The patient was placed into the lateral position and a midline posterior cervical incision made. Initially, the insertion point of the electrodes was the spinous process of $\mathrm{C} 1$, passing superior and laterally, using a curved Tuohy needle and an image intensifier 
to aid positioning. This method evolved over time so that implantation level was aimed at the greater occipital nerve as it emerged superior to the nuchal line. In this amended technique, the electrode was passed using a blunt plastic tube to limit the risks of the electrode tip being tunneled too close to the skin. The evolution of surgical technique occurred in response to adverse events such as recruitment of neck muscles during stimulation or erosion of the electrode tip through the scalp. Given that both techniques target the same nerve it is felt unlikely that the implant technique would directly account for changes in efficacy. Electrodes were looped and anchored to cervical fascia and then tunneled to a lateral cervical or infraclavicular skin crease intermediate incision. An infraclavicular or abdominal incision was made (according to patient preference) and a pocket formed into which the IPG was placed. Electrodes were tunneled to the intermediate incision site where a pair of extension leads were connected. Silicone sheaths were used to protect lead connections. Topical gentamicin was introduced around the pocket prior to closure. Our unit did not employ trial stimulation as it was felt that the current evidence to support its use is outweighed by the risks of extra surgical procedures.

Patients were provided with remote controls allowing them to adjust their stimulation amplitude but were asked to use continuous stimulation where possible. Polarity of the electrodes was adjusted during follow up visits to ensure comfortable bilateral paresthesia in the occipital region. Stimulation settings and changes were recorded at each visit. Medications were changed as needed at the discretion of the headache specialist.

\section{Data Collection}

Primary outcome measure was the change in mean daily attack frequency. Secondary measures included: the clinical response to ONS (defined as a more than 50\% reduction in 
attack frequency), changes in attack severity and duration, headache specific disability scores, quality of life scores and affect scores.

Outcome data was collected and recorded prospectively. Prospective headache diaries were kept for one month prior to implant and then two weeks prior to each follow-up visit. All patients were shown how to keep a paper diary recording the duration and pain intensity of their attacks and asked to record all daily attacks. Patients were seen at three-monthly intervals post-implant for one year and at least six to twelve monthly thereafter depending on clinical condition. Data was collected on demographics, diagnosis, treatments, attack frequency, severity and duration, headache disability scores, quality of life scales, affective scores, ONS settings and adverse events. Although specific tools for measuring the associated disability of SUNHA have yet to be validated, Migraine Disability Assessment Scores (MIDAS) and Headache Impact Test-6 (HIT-6) scores were conducted. These scores have been used extensively in the assessment of primary headache disorders and previously used to monitor response of trigeminal autonomic cephalalgias, including SUNHA, to ONS ${ }^{18 \text {, }}$ 23. Euro-QoL (EQ5D), Short Form-36 Questionnaires (SF36), Beck Depression Inventory (BDI-II), Hospital Anxiety (HAD-A) and Depression (HAD-D) scores were used to monitor quality of life and affect pre-and post-implant.

Data was collected prospectively from 2007 until 2015 and entered onto a clinical database (Microsoft Excel, Microsoft Corporation, Redmond, WA, USA). 


\section{Statistics}

All statistical analyses were conducted using IBM SPSS Statistics version 22 (IBM Corp. Int.). A last observation carried forward technique was used in the case of missing data. Descriptive statistics were summarized as appropriate. Data is presented as mean \pm standard deviation or range where appropriate. Paired and independent t-tests were used to compare treatment effect. All statistical tests were two-sided with a significance level of $95 \%$.

\section{RESULTS}

\section{Patient demographics}

Thirty-one patients (14 male) with SUNHA were implanted between August 2007 and December 2013. Provisional data from some of these patients had been previously included in a publication from $2014^{23}$. Patient demographics and baseline headache characteristics are shown in Table 1. Mean age at implant was 48.9 years (range 20-74). Seventy-seven percent were chronic from onset with mean chronic phase duration of 6.3 years (range 2-21 years). Patients had tried a mean of 7.8 preventative medications prior to ONS (range 5-11) summarized in Supplementary Table 1.

Sixteen patients $(51.6 \%)$ reported other headache phenotypes in addition to SUNHA: nine with chronic migraine, three with both chronic migraine and chronic cluster headache, two with hemicrania continua, one with chronic migraine and hemicrania continua, and one with chronic cluster headache (Table 1). All kept separate diaries for each phenotype throughout follow-up.

\section{Whole cohort}


Mean follow-up for all patients was 44.9 months (range 13-89 months). At final follow-up, mean daily attack frequency had reduced by $69 \%( \pm 38.2 \%)$ from 98.5 to 31.2 attacks $(\mathrm{p}=0.012)$. Figure 1a shows the change in daily attack frequency over the follow-up period. A $50 \%$ or more reduction in daily attack frequency was observed in $77 \%(n=24)$ of patients. Twelve patients were pain free at final-follow up with a mean complete remission time of 36.5 months (10-78). Significant improvements were also seen in attack severity (5.5 points on verbal response scale [VRS]; $\mathrm{p}<0.001)$ and attack duration $(64 \% ; \mathrm{p}=0.001)$ (Table 2). At final follow-up, reductions in both MIDAS (40.6 points; p=0.053) and HIT-6 (4.7 point; $\mathrm{p}=0.017$ ) were observed although only HIT-6 reduction was significant. Affect scores showed a significant reduction in HAD-D but non-significant reductions in HAD-A and BDIII. The SF36-P, SF36-M and EQ-5D all showed non-significant improvement but EQ-VAS showed significant improvement of 13.1 points $(\mathrm{p}=0.028)$ (Table 3). Mean patient estimated improvement across all patients was $72 \%( \pm 34.0)$

\section{Short lasting unilateral neuralgiform headache attacks alone}

In the 15 patients with SUNHA alone, the mean follow-up was 44.2 months (range 13-81). A 50\% reduction in daily attack frequency was seen in $67 \%(\mathrm{n}=10)$ at final follow-up. Mean daily attack frequency reduced by $61.9 \%( \pm 43.1)$ from 152.3 to $57.5(\mathrm{p}=0.025)$. Figure $1 \mathrm{~b}$ shows the reduction in daily attack frequency over the follow-up period in this cohort. Reductions were also seen in attack intensity (5.0 points on VRS; $\mathrm{p}=0.001)$ and attack duration $(68 \% ; \mathrm{p}=0.011)$. Significant improvements were seen in MIDAS (71.4 points, $\mathrm{p}=0.023)$ but not in HIT-6. Affect scores showed significant improvements in HAD-A, HAD-D and BDI-II (Table 3) but no significant changes were seen in any of the other quality of life scores. Mean patient estimated improvement was $70 \%$ $( \pm 38.4)$. 


\section{Multiple phenotypes including SUNHA}

In the 16 patients with multiple headache phenotypes, the mean follow-up was 45.6 (range 15-89). A $50 \%$ reduction in daily attack frequency was seen in $88 \%(n=14)$ at final follow-up. Mean daily attack frequency reduced from 55.3 to 9.8 , a change of $75 \%( \pm 32.9 ; \mathrm{p}=0.001)$. At three-months post implant there was a significant difference in the reduction in attack frequency between those with and without multiple phenotypes (figure $1 \mathrm{~b}$ and Table 3 ), at all other time points, response was comparable in both cohorts. Significant changes were observed in attack severity (4.4 points on VRS; $\mathrm{p}=0.002$ ) and attack duration (57\%; $\mathrm{p}=0.003)$. Although reductions were seen in MIDAS (11.5 points) and HIT-6 (4.0 points) only that in HIT-6 was significant $(\mathrm{p}=0.032)$. No significant changes were seen in any of the affect or quality of life scores (Table 4). Mean patient estimated improvement was $75 \%( \pm 30.3)$.

In those whose SUNHA responded to ONS, 6/11 chronic migraines, 1/3 chronic cluster headaches and 2/3 hemicrania continua also showed improvement (defined as a more than $50 \%$ reduction in daily attack frequency for $\mathrm{CCH}$ and a more than $30 \%$ reduction in moderate-to-severe headache days for chronic migraine and hemicranias continua). In those SUNHA non-responders, $1 / 2$ of the co-existent chronic migraines responded to ONS.

\section{Concomitant drug use}

Twenty-five patients were taking preventative medication at time of implant (mean two drugs). At follow-up six patients previously on preventative drugs had stopped all medication and ten had reduced the dose or number of drugs taken from baseline. In responders to ONS, 18/24 had medications started during follow-up, however, in only three cases was this 
medication for SUNHA and in one patient medication for SUNHA was started after the ONS removed.

\section{Time to effect and recurrence of attacks}

The mean time of all patients to reach a 50\% improvement was 5.7 months (range 1-19.0). In responders this time was 5.3 months (range 1-12.0). A significant difference was seen in daily attack frequency between baseline and three months in responders ( 85.3 attacks; $\mathrm{p}=0.031)$ but no such change was observed between month three and any other time point.

Twelve patients had their ONS turned off for a period of time (median 8months, range 2-60 months) -8 due to battery depletion, two due to lack of efficacy, one due to explantation and one due to technical reasons. In ten of these, SUNHA attacks worsened with ONS off within a mean time of 1.2 months (range 1-3).

\section{Stimulation settings}

All patients were encouraged to use continuous stimulation. A range of settings was used to achieve the widest area of occipital paresthesia possible. As a group, the range for amplitude was $0.20-4.65$ volts (mean 1.4 volt), pulse width $294-480 \mu$ s (mean $441.5 \mu \mathrm{s}$ ) and frequency 30-170 Hz (mean $78.3 \mathrm{~Hz})$.

\section{Adverse events}

A total of 35 adverse events involving 20 patients were recorded (Table 5). Events were recorded as "hardware related" if they involved malfunction of a device component, 
"biological" if they involved pain or biological reactions to the device and "stimulationassociated" if they were related to level of stimulation. No lead migration or electrode erosion was recorded in the group. One patient (3\%) suffered a minor wound infection requiring medical management. Only one patient elected to have the device removed due to lack of efficacy after two-years.

A total of 11 events required surgical intervention, the majority being revisions of the ONS system to rechargeable batteries.

\section{DISCUSSION AND CONCLUSIONS}

This cohort is the largest reported for ONS in intractable chronic SUNHA. In line with previous open-label series of chronic cluster headache and the single series of SUNHA patients, we suggest that ONS may be a beneficial treatment with sustained effects for refractory patients ${ }^{18-23}$. Our series of 31 complex patients reported a significant reduction in daily attack frequency of $69 \%$ with 12 patients (39\%) recording complete pain freedom at follow-up. Overall, $77 \%$ of patients had a more than $50 \%$ reduction in daily attack frequency with ONS. Significant improvements were seen in the headache-specific disability score HIT-6 and quality of life component EQ-VAS but not in other quality of life measures or MIDAS.

Occipital nerve stimulation was first used for presumed occipital neuralgia but has since been used for intractable chronic migraine and chronic trigeminal autonomic cephalalgias ${ }^{28,} 29$. Controlled trials in chronic migraine have shown limited and conflicting evidence of efficacy 
15-17. Although controlled trials of ONS in trigeminal autonomic cephalalgias have not been completed, reviews of the open label series of chronic cluster headache report a clinical response in $67 \%$ of patients ${ }^{29}$. In the single published series of ONS for refractory SUNHA a cohort of nine patients was reported using headache load as primary outcome ${ }^{23}$. In that small series, $89 \%$ of patients recorded a headache load reduction of at least $50 \%$ at a median of 38 months.

Clinically and statistically important reductions in HIT-6, HAD-D and EQ5D-VAS scores were seen following ONS but no significant improvements were seen in other quality of life measures or migraine specific scores. Reasons for this may include sample size, long duration of chronic pain (7 years), co-existent headache disorders and the usefulness of generic scales in measuring quality of life in headache patients. The cohort continued to suffer a mean total of nearly 90 minutes of pain a-day, which, although a significant reduction from the pre-ONS value of 9.5 hours is still a considerable burden. In those with multiple headache types not all other headaches improved. The impact of continued disabling chronic headaches in this sub-population may negatively influence quality of life at follow-up and this is supported by a more favorable quality of life profile in those with SUNHA alone than in multiple phenotype patients (Table 3).

In keeping with previous ONS series for other headache conditions our cohort reported an average delay of six months before reporting a clinical response and a return of attacks after an average of one month when stimulation was stopped $16,18-20,22,23,29$. The delay and reversibility of effect reflects the slow but reversible neuroplastic response proposed to underlie ONS treatment. 
The adverse event profile of ONS for headache has been a major cause of criticism regards the treatment. Previous ONS series reported high rates of lead migration (7-50\%), lead fracture (10-15\%) and infection (10-24\%) ${ }^{15,18-21,30}$. Our cohort has a favorable adverse event profile with no episodes of lead migration, fracture or erosion and only one patient (3\%) suffering a superficial wound infection requiring medical management only. The major need for surgical intervention was battery replacement and with developments in neuromodulation technology leading to the use of longer lasting rechargeable batteries the rates of battery replacement should decrease in the future.

The major weakness of this study is the lack of placebo or blinded stimulation. However, this has been an issue throughout ONS research and with such a rare syndrome as SUNHA it is unlikely a large randomized trial will ever be completed. Although there is undoubtedly a placebo effect in headache treatment it is unlikely our findings are explained by placebo alone. The intractable nature of the group, the delay to clinical response in keeping with other trigeminal autonomic cephalalgia cohorts, the stable long-term response and the return of attacks when stimulation is stopped would argue against a pure placebo response. It has also been reported that the placebo response rates for ONS in migraine is low $(6 \%, 17 \%$ and $20 \%)$ and there is no evidence to suggest placebo response is different in other headache conditions $15-17$

The patient group included a high proportion of patients (52\%) with multiple chronic headache phenotypes. These patients had all been carefully phenotyped by headache specialists and patients were able to differentiate the different types recorded them in separate diaries to allow outcomes to be compared. Although this percentage may seem excessive, other SUNHA cohorts have also described similar patterns ${ }^{2,31}$. It is speculated that those with 
multiple headaches fare worse with treatment, however, our data, all be it limited, does not support this. Occipital nerve stimulation has been used to treat a number of headache conditions and we view it as a potentially useful treatment to those with multiple headache types as a single treatment modality can improve multiple conditions. More work is needed on the outcomes of such complex patients to all treatments not just ONS, but our data suggests that they should not be deprived of ONS on the assumption that complex patients do not respond well.

Strengths of the study include the sample size, prolonged follow-up, prospective collection of data and the clinical relevance of the data. The cohorts were not selected by vigorous study inclusion criteria but were patients in a specialist center implanted due to clinical need when ONS was only available as a last-line treatment option. The cohort will be similar to patients in other units conducting implants for headache conditions and so our findings should be widely and clinically applicable.

Microvascular decompression and deep brain stimulation have also been reported in openlabel series to have efficacy in SUNHA disorders of around $75 \%$, similar to the response rate in this ONS series ${ }^{12,29,32-34}$. The surgical experience of the team, the invasiveness of surgery, the associated risks, the need for implanted hardware and cost of treatment will all influence treatment choice. Bearing these factors in mind, our current pathway is that patients with intractable SUNHA first undergo microvascular decompression (if there is evidence of trigeminal neurovascular contact on neuroimaging), and ONS be reserved for those with no neurovascular compromise or who fail to respond to microvascular decompression. This order is based on our own clinical experience, technical expertise and treatment availability. 
Our series suggests that ONS may provide a significant and sustained reduction in attack frequency in intractable short-lasting unilateral neuralgiform headache attacks even after four years follow-up. Adverse event rates are low when implants are conducted in specialist centers. However, given the invasiveness and cost of treatment, ONS should be reserved for those who fail all other appropriate treatment options. 


\section{ACKNOWLEDGEMENTS}

We would like to thank our Headache Specialist Nurses, especially Miss Susie Lagrata, for their help with completion of the clinical database and management of the patients. We also thank the patients and their families for their help with this project.

\section{CONTRIBUTORS}

SM: recruitment of subjects, analysis and interpretation of data, drafting and revision of manuscript.

LW: performed surgery and manuscript revision

MSM: study concept, recruitment of subjects, interpretation of data and manuscript revision.

\section{COMPETING INTERESTS}

SM has received educational and travel grants from St Jude Medical and Medtronic. LW declares no competing interests. MSM serves on the advisory board for Allergan, St Jude Medical and Medtronic and has received payment for the development of educational presentations from Allergan, Merck Sharpe and Dohme Ltd, Medtronic and electroCore.

\section{ETHICS APPROVAL}

Ethical approval for the collection, storage and publication of data was obtained from subjects undergoing ONS implantation. Ethic board approval for data collection and publication was granted by Northwick Park Hospital Research Ethics Committee, Hampstead, London, UK. 


\section{Clinical implications}

- Short lasting unilateral neuralgiform headache disorders are intensely painful and highly disabling. A significant minority of these patients are intractable to medical treatments

- Occipital nerve stimulation (ONS) is associated with sustained reduction in daily attack frequency, severity and duration over a 4-year period in some patients with intractable short lasting unilateral neuralgiform headache disorders

- When undertaken by highly specialised centres, the complication rates associated with ONS implantation are relatively low 


\section{REFERENCES}

1. Headache Classification Committee of the International Headache Society. The International Classification of Headache Disorders, 3rd edition (beta version). Cephalalgia. 2013; 33: 629-808.

2. Cohen AS, Matharu MS and Goadsby PJ. Short-lasting unilateral neuralgiform headache attacks with conjunctival injection and tearing (SUNCT) or cranial autonomic features (SUNA)--a prospective clinical study of SUNCT and SUNA. Brain. 2006; 129: 2746-60.

3. Cohen AS. Short-lasting unilateral neuralgiform headache attacks with conjunctival injection and tearing. Cephalalgia. 2007; 27: 824-32.

4. Piovesan EJ, Siow C, Kowacs PA and Werneck LC. Influence of lamotrigine over the SUNCT syndrome: one patient follow-up for two years. Arq Neuropsiquiatr. 2003; 61: 6914.

5. Porta-Etessam J, Benito-Leon J, Martinez-Salio A and Berbel A. Gabapentin in the treatment of SUNCT syndrome. Headache. 2002; 42: 523-4.

6. Dora B. SUNCT syndrome with dramatic response to oxcarbazepine. Cephalalgia. 2006; 26: 1171-3.

7. Matharu MS, Boes CJ and Goadsby PJ. SUNCT syndrome: prolonged attacks, refractoriness and response to topiramate. Neurology. 2002; 58: 1307.

8. Arroyo AM, Duran XR, Beldarrain MG, Pinedo A and Garcia-Monco JC. Response to intravenous lidocaine in a patient with SUNCT syndrome. Cephalalgia. 2010; 30: 110-2.

9. Matharu MS, Cohen AS and Goadsby PJ. SUNCT syndrome responsive to intravenous lidocaine. Cephalalgia. 2004; 24: 985-92.

10. Porta-Etessam J, Cuadrado ML, Galan L, Sampedro A and Valencia C. Temporal response to bupivacaine bilateral great occipital block in a patient with SUNCT syndrome. $J$ Headache Pain. 2010; 11: 179.

11. Black DF and Dodick DW. Two cases of medically and surgically intractable SUNCT: a reason for caution and an argument for a central mechanism. Cephalalgia. 2002; 22: 201-4.

12. Sebastian S, Schweitzer D, Tan L and Broadley SA. Role of trigeminal microvascular decompression in the treatment of SUNCT and SUNA. Curr Pain Headache Rep. 2013; 17: 332.

13. Miller S, Akram H, Lagrata S, Hariz M, Zrinzo L and Matharu M. Ventral tegmental area deep brain stimulation in refractory short-lasting unilateral neuralgiform headache attacks. . Brain. 2016; 139: 2631-40.

14. Schoenen J, Di Clemente L, Vandenheede M, et al. Hypothalamic stimulation in chronic cluster headache: a pilot study of efficacy and mode of action. Brain. 2005; 128: 9407.

15. Lipton R, Goadsby PJ, Cady R, et al. PRISM study: Occipital nerve stimulation for treatment-refractory migraine. Cephalalgia. 2009; 29: 30.

16. Silberstein SD, Dodick DW, Saper J, et al. Safety and efficacy of peripheral nerve stimulation of the occipital nerves for the management of chronic migraine: results from a randomized, multicenter, double-blinded, controlled study. Cephalalgia. 2012; 32: 1165-79.

17. Saper JR, Dodick DW, Silberstein SD, McCarville S, Sun M and Goadsby PJ. Occipital nerve stimulation for the treatment of intractable chronic migraine headache: ONSTIM feasibility study. Cephalalgia. 2011; 31: 271-85.

18. Burns B, Watkins L and Goadsby PJ. Treatment of intractable chronic cluster headache by occipital nerve stimulation in 14 patients. Neurology. 2009; 72: 341-5. 
19. Fontaine D, Christophe Sol J, Raoul S, et al. Treatment of refractory chronic cluster headache by chronic occipital nerve stimulation. Cephalalgia. 2011; 31: 1101-5.

20. Magis D, Gerardy PY, Remacle JM and Schoenen J. Sustained effectiveness of occipital nerve stimulation in drug-resistant chronic cluster headache. Headache. 2011; 51: 1191-201.

21. Palmisani S, Al-Kaisy A, Arcioni R, et al. A six year retrospective review of occipital nerve stimulation practice--controversies and challenges of an emerging technique for treating refractory headache syndromes. J Headache Pain. 2013; 14: 67.

22. Miller S, Watkins L and Matharu M. Treatment of intractable chronic cluster headache by occipital nerve stimulation: a cohort of 51 patients. Eur J Neurol. 2017; 24: 38190 .

23. Lambru G, Shanahan P, Watkins L and Matharu MS. Occipital Nerve Stimulation in the Treatment of Medically Intractable SUNCT and SUNA. Pain Physician. 2014; 17: 29-41.

24. Headache Classification Subcommittee of the International Headache Society. The International Classification of Headache Disorders, 2nd edn. Cephalalgia. 2004; 24: 1-160.

25. Antonaci F, Costa A, Ghirmai S, Sances G, Sjaastad O and Nappi G. Parenteral indomethacin (the INDOTEST) in cluster headache. Cephalalgia. 2003; 23: 193-6.

26. Williams MH and Broadley SA. SUNCT and SUNA: clinical features and medical treatment. J Clin Neurosci. 2008; 15: 526-34.

27. Kinfe TM, Schuss $\mathrm{P}$ and Vatter H. Occipital nerve block prior to occipital nerve stimulation for refractory chronic migraine and chronic cluster headache: Myth or prediction? Cephalalgia. 2015; 35: 359-62.

28. Weiner RL and Reed KL. Peripheral neurostimulation for control of intractable occipital neuralgia. Neuromodulation. 1999; 2: 217-21.

29. Magis D and Schoenen J. Advances and challenges in neurostimulation for headaches. Lancet Neurol. 2012; 11: 708-19.

30. Sharan A, Huh B, Narouze S, et al. Analysis of Adverse Events in the Management of Chronic Migraine by Peripheral Nerve Stimulation. Neuromodulation. 2015; 18: 305-12.

31. Lambru G, Shanahan P and Matharu M. Exacerbation of SUNCT and SUNA syndromes during intravenous dihydroergotamine treatment: A case series. Cephalalgia. 2015.

32. Leone M, Franzini A, D'Andrea G, Broggi G, Casucci G and Bussone G. Deep brain stimulation to relieve drug-resistant SUNCT. Ann Neurol. 2005; 57: 924-7.

33. Lyons MK, Dodick DW and Evidente VG. Responsiveness of short-lasting unilateral neuralgiform headache with conjunctival injection and tearing to hypothalamic deep brain stimulation. J Neurosurg. 2009; 110: 279-81.

34. Bartsch T, Falk D, Knudsen K, et al. Deep brain stimulation of the posterior hypothalamic area in intractable short-lasting unilateral neuralgiform headache with conjunctival injection and tearing (SUNCT). Cephalalgia. 2011; 31: 1405-8. 
